\title{
Claves para el desarrollo de la comunicación corporativa en los sectores tradicionales
}

Dra. Rocío Blay Arráez | rblay@uji.es

UNIVERSITAT JAUME I

Doctora en Ciencias de la Comunicación por la Universitat Jaume I de Castellón. Licenciada en Ciencias de la Información y Postgrado en Gestión Publicitaria en la UCM. Actualmente desarrolla su actividad docente en la Universitat Jaume I, dirigiendo el Grado de Publicidad y RRPP e investigando en los ámbitos de la gestión estratégica de la comunicación corporativa y en creatividad publicitaria.

\section{Dra. Maria Teresa Benlloch Osuna | mbenlloch@uji.es}

UNIVERSITAT JAUME I

Doctora en Ciencias de la Comunicación por la Universitat Jaume I de Castellón. Licenciada en Administración de Empresas. Actualmente combina actividad docente e investigadora en la Universitat Jaume $I$ y en otros cursos y maestrías, en los ámbitos de la gestión estratégica de intangibles, concretamente de la gestión estratégica de la comunicación financiera y de la marcas territoriales.

\section{Dr. Guillermo Sanahuja Peris | gsanahuj@uji.es}

UNIVERSITAT JAUME I

Doctor en Ciencias de la Comunicación. Licenciado en Publicidad, Máster en Dirección de Marketing y Comunicación por IDE-CESEM y Máster Nuevos Procesos en Comunicació. Ejerce como consultor en SANAHUJA \& GIMENO y como profesor asociado en la UJI.

\section{Resumen}

Esta investigación se propone ofrecer claves que propicien el desarrollo de la comunicación corporativa en los sectores tradicionales, sectores con un peso muy relevante en nuestra economía. Tras profundizar en los pilares teóricos del corporate y en la casuística de estos sectores, se desarrolla una metodología cualitativa en la que han participado empresarios de éstos sectores, representantes de asociaciones sectoriales y profesionales del ámbito de la comunicación. Las conclusiones arrojan una serie de sugerencias vinculadas a tres dimensiones cuyo objeto es instalar la cultura de la comunicación en estas organizaciones, conseguir una cooperación entre las distintas empresas para construir marcas territorio potentes y favorecer la relación de confianza y eficacia entre agencias y organizaciones.

Palabras clave: comunicación, corporate, sectores empresariales tradicionales; 
Abstract: The purpose of this research is to offer clues which contribute to the development of communication in the traditional sectors. After an in depth study of the corporate's theoretical pillars and these sectors' casuistry, it's been developed a qualitative methodology with the involvement of its professionals, representatives of the sectorial associations and other professionals in the communication field. The conclusions include a list of suggestions linked to three dimensions that have as an objective to install the communication culture, achieving a cooperation between the diferent enterprises in order to build strong territorial brands and to help the trusting and efficient relationship between agencies and organisations.

Key words: communication, corporate, traditional business sectors. 


\section{Introducción: El interés de esta investigación}

Esta investigación que centra su foco en la Comunidad Valenciana presenta unas conclusiones que son extrapolables a otros sectores tradicionales y a otros territorios por la homogeneidad en sus estructuras, de unos sectores empresariales que mantienen una fuerte penetración a lo largo y ancho de la geografía española.

Los sectores tradicionales de la Comunidad Valenciana (agroalimentación, calzado, cerámica, iluminación, juguete, mueble y textil) son auténticos cluster ${ }^{1}$ generadores de empleo y riqueza. De hecho, por su alto grado de concentración geográfica, hay poblaciones donde más del 50\% del empleo viene generado por ellos. No obstante, atraviesan desde hace años por una situación crítica (Pla Barber, J. y Otros, 2007). La Cámara de Comercio de Valencia ya publicó en 2004 un informe elaborado por la Universidad Politécnica (Cámara Oficial de Comercio, Industria y Navegación de Valencia, 2004) donde retrataban la difícil situación que estaban atravesando y reclamaban por parte de las administraciones públicas iniciativas concretas para frenar la inercia de cierre de empresas y pérdida de empleo.

Este artículo, como continuación de anteriores comunicaciones publicadas entre 2006 y 2010 (Blay Arráez, R. y Benlloch Osuna, Ma.T, 2006, 2007, 2010; y Benlloch Osuna, Ma T y López Lita, R, 2005, 2006), considera que el análisis de la gestión de la comunicación corporativa en estos sectores y la importancia que se le atribuye es clave para revelar las carencias de estas organizaciones en el contexto actual de la sociedad de la información. Una vez conocido éste estado de la comunicación, la investigación propone planes que permitan avanzar en la gestión de la comunicación corporativa y en la creación de marcas fuertes y competitivas como factor clave para el futuro de éstos sectores.

\section{El marco teórico}

\subsection{La función de la comunicación corporativa como activo empresarial estratégico.}

En la actualidad muchas corporaciones ya han tomado un nuevo rumbo en la gestión que tiene que ver más con una nueva sensibilidad empresarial, donde se pretende revertir parte del beneficio que tienen las empresas en el territorio donde desarrolla su actividad, para conjugar los intereses empresariales con los de los públicos con los que se relaciona (Costa, 2008). La aceptación general de la responsabilidad pública que tienen las organizaciones es un hecho vital y existe ya una consciencia de la necesidad que tiene la empresa de demostrar un comportamiento socialmente responsable, con el objeto de obtener ahora sí la

\footnotetext{
${ }^{1}$ Los clusters, son grupos internamente homogéneos, pero distintos entre sí, bien por agrupación de unidades más pequeñas o por división de segmentos mayores, se ha aplicado en marketing especialmente en segmentación de mercados. Un cluster en el mundo industrial es una concentración de empresas relacionadas entre sí, en una zona geográfica relativamente definida, que conforman en sí mismas, un polo productivo especializado con ventajas competitivas.
} 
plena confianza de la opinión pública. Esta confianza es el estadio final de un complejo proceso valorativo. El inicio de este proceso y la vía más directa para avanzar consiste en generar un reconocimiento positivo del público a patir de canales de comunicación constantes y bidireccionales (Capriotti, 2009) con los públicos, y fomentar así, la participación de los ciudadanos en el destino de la organización.

Dentro de todos estos cambios en la filosofía empresarial también debe ser apuntada la revolución de los servicios, de la que habla Joan Costa (2003), que surge de la sustitución de las personas a las máquinas. En esta nueva perspectiva, el "cara a cara" ya no es el del consumidor con el producto inerte. Es el cliente con el empleado, es decir, con la empresa, en la medida que éste la representa explícitamente y es parte de ella. Con las personas en primer plano en la cultura de servicio, las empresas redescubren los recursos humanos, y con ello, se impone la exigencia de programas de calidad, de cultura organizacional con la gestión de carreras, y la comunicación interna como agente integrador.

Al mismo tiempo, se da ascenso del "corporate", o función estratégica de la comunicación corporativa (Villafañe, 1999) ya que toda esta serie de transformaciones que ha arrastrado la revolución de los servicios puso el acento en la institución y en la estrategia global del negocio. Así, los productos se han quedado circunscritos al campo del consumo mientras que la imagen y la marca ocupan el primer plano. En este salto evolutivo del "corporate" primero fue la reafirmación de la identidad corporativa - la empresa como realidad única, total e irrepetible- y a continuación la asunción de un espíritu institucional en la gestión integrada de los recursos. "Es la irrupción revolucionaria de los intangibles y los valores" tal y como señala Pizzolante Negrón (2006).

Dentro de este paradigma de la nueva empresa, la comunicación corporativa deberá gestionar la totalidad de técnicas de comunicación y recursos intangibles de los que dispone una organización para llegar efectivamente a sus públicos (Garrido, 2001). En esta gestión jugará un papel fundamental la identidad corporativa en primera instancia y el objetivo último será que la imagen corporativa sea una síntesis fidedigna de la identidad corporativa (Costa, 2004). Para alcanzar este objetivo habrá que gestionar a su vez tanto el comportamiento, la cultura corporativa y la marca bajo el concepto marca experiencia. Finalmente una vez alcanzadas estas metas se pretende convertir la imagen positiva en reputación corporativa, fin último de la comunicación corporativa (Villafañe, 2004). La relevencia de estos recursos intangibles como fuente de valor de las organizaciones y elemento decisor en mercados homogéneos convierten, por tanto, a la función de la comunicación corporativa en un activo empresarial estratégico.

\subsection{Particularidades de la gestión de la comunicación en pymes de la Comunidad} Valenciana. 
Los sectores objeto de estudio de esta investigación son mayoritariamente pymes y esta peculiaridad condiciona dicha gestión. Además, se profundiza en el anunciante valenciano, revisando si también posee particularidades dignas de reseña. Por tanto, la aproximación será desde lo más general, las pymes, hasta los clusters concretos sobre los que se está investigando y que como ya se ha concretado en el punto anterior son agroalimentación, calzado, cerámica, iluminación, juguete, mueble y textil.

\subsubsection{Investigaciones previas sobre pymes de la Comunidad Valenciana}

En relación a la caracterización específica de los sectores tradicionales de la Comunidad Valenciana se debe destacar que existen pocas investigaciones específicas sobre la gestión que hacen de su comunicación publicitaria y menos, sobre la corporativa. Sí existen algunos informes sectoriales o investigaciones académicas que hacen mención a algunos de los clusters pero siempre tratan el tema de manera indirecta. No obstante, aglutinando toda la información aparecida en diferentes fuentes (Cámara Oficial de Comercio, Industria y Navegación de Valencia, 2004) se puede concluir que estos sectores, pese a tener un número muy elevado de marcas y de anunciantes, generan un volumen de inversión publicitaria muy pequeño. Además se produce una elevada concentración de las acciones de comunicación en muy pocos soportes, principalmente en revistas especializadas, evidenciando que la una técnica de comunicación utilizada es la publicitaria, en detrimento de otras como la comunicación interna, la comunicación de crisis, las relaciones informativas, la comunciación financera o el patrocino; y que no existen programas integrales de comunicación corporativa.

\subsubsection{Características básicas de la gestión de la comunicación en las pymes españolas}

Las características básicas de la gestión de la comunicación que realizan las pymes (Carrillo, 2005) y que vienen condicionadas, por el ajuste que hacen a su volumen de negocio y a sus posibilidades, son las siguientes:

a.- Las pymes comunican y conocen las herramientas tradicionales de la comunicación comercial, especialmente publicidad, promociones, marketing directo y comunicación en el punto de venta, y aunque desconocen las últimas tendencias en la materia, sí están familiarizadas con la gestión de la comunicación publicitaria o comercial.

b.- Son muy exigentes con la rentabilidad de la comunicación, aunque esto lo miden solo en términos de venta.

c.- Al ser empresas de tamaño pequeño, perciben mayor riesgo a hacer alguna acción de comunicación que no obtenga resultados.

d.- El punto fuerte de sus acciones de comunicación es el contacto personal que pueden mantener con cada colectivo. 
e.- El control personal de su negocio les lleva a querer ser autosuficientes y no contar con profesionales externos.

f- El escaso conocimiento de lo que significa la comunicación empresarial, lleva a que en la mayor parte de los casos, lo relacionen exclusivamente con publicidad y ofertas comerciales.

g.- Aquellos que apuestan por llevar a cabo acciones publicitarias, apuestan por mensajes racionales apoyados, en la mayoría de casos, en el propio producto.

\section{Objetivo, hipótesis, metodología y trabajo de campo}

\subsection{Objetivo}

Esta investigación tiene como objetivo la propuesta de una serie de claves que propicien el desarrollo de la comunicación corporativa en los sectores tradicionales valencianos atendiendo a tres dimensiones o niveles micro, meso y macro.

\subsection{Hipótesis}

De acuerdo con los precedentes esta investigación se plantea la siguiente hipótesis: Los denominados sectores empresariales tradicionales de la Comunidad Valenciana deben aplicar de manera urgente, una serie de medidas para la necesaria evolución en su modelo de comunicación corporativa, con el fin de potenciar su competitividad y garantizar su futuro empresarial. Las palancas de cambio están asociadas a la instalación de la cultura de la comunicación en estos sectores, la oportuna cooperación entre las distintas empresas para construir marcas territorio potentes y la fijación de una relación de confianza y eficacia entre agencias y organizaciones.

\subsection{Metodología}

Para llevar a cabo esta investigación se ha elegido el modelo de investigación propuesto por el catedrático Rafael López Lita (2001) en la publicación Las Agencias de Publicidad: evolución y posicionamiento futuro. Así, el presente trabajo se articula en tres grandes etapas que se explican a continuación:

\subsubsection{Marco Teórico: fase documental.}

Se presenta todo el marco teórico y documental que sienta el punto de partida de la investigación.

3.3.2. Marco Empírico: trabajo de campo. 
La siguiente etapa de la investigación consiste en el desarrollo del trabajo de campo. A continuación se describe la metodología empleada, el universo, la muestra seleccionada y las técnicas de investigación empleadas, todas ellas de corte cualitativo, a lo largo de las tres sub-etapas del trabajo de campo que concluyeron en 2010. Concretamente, en el trabajo de campo I se desarrollan 7 entrevistas en profundidad, en el trabajo de campo II, 28 entrevistas en profundidad y en el trabajo de campo III se realizan dos grupos focales. Todas ellas basadas en técnicas de investigación cualitativa (Álvarez-Gayou Jurgenson, 2003)

Trabajo de campo I y II

Para alcanzar el objetivo propuesto a trabajar con estos sectores, se establecieron dos grupos potenciales a los que investigar:

- En un primer lugar (equivale al trabajo de campo I), se entrevistó a los Secretarios Generales de las Asociaciones Empresariales representativas de los siete sectores tradicionales de la Comunidad Valenciana seleccionados.

- En un segundo lugar (equivale al trabajo de campo II), a un número determinado de empresas de cada uno de los sectores objeto de estudio, donde se estrevistó a los gerentes o directores generales.

- El universo de estudio de esta investigación está formado por 8.313 empresas enmarcadas dentro de los siete sectores tradicionales de la Comunidad Valenciana -agroalimentación, calzado, cerámica, iluminación, juguete, mueble y textil - según fuentes consultadas en el año 2007. Una vez definido el universo, tanto a nivel cualitativo como cuantitativo, se describe la selección de la muestra que los representa en este trabajo de campo I y II. A pesar de que se trata de una muestra cualitativa de conveniencia, ante el gran número de empresas que conforman se plantea la necesidad de realizar dos tipos de aproximaciones, una a través de las asociaciones que hablan en nombre de todos sus asociados y otra, directamente con las empresas de los sectores seleccionados.

- $1^{\text {a }}$ Muestra del trabajo de campo I. Asociaciones representantes de los sectores seleccionados.

En este cuadro que a continuación se presenta, se refleja de cada sector, la asociación y la persona a la que se ha entrevistado a finales del 2006, además se añaden como datos relevantes el ámbito de actuación de la misma, ya que en algunas ocasiones representan sólo a la Comunidad Valenciana y en otras, son asociaciones que aunque su principal territorio está en la comunidad cubren el ámbito nacional. Como dato cuantitativo se aporta el número de asociados que tienen y por tanto, al número de empresas a las que representan.

Cuadro I. Muestra del trabajo de campo I. 


\begin{tabular}{|c|c|}
\hline SECTOR TRADICIONAL CV & ASOCIACIÓN EMPRESARIAL \\
\hline 1.- AGROALIMENTACIÓN & $\begin{array}{l}\text { FEDACOVA (Federación empresarial de agroalimentación de la } \\
\text { Comunidad Valenciana) } \\
\text { D. José Granell Pascual (Secretario General) } \\
\text { ÁMBITO ACTUACIÓN: Comunidad Valenciana. No ASOCIADOS: } 32 \\
\text { Asociaciones que representan } 2100 \text { empresas. }\end{array}$ \\
\hline 2.- CALZADO & $\begin{array}{l}\text { AICE (Asociación de Industriales de calzado de Elche) } \\
\text { FICE (Fed.de Industriales de calzado de la Comunidad Valenciana) } \\
\text { D. Pedro Méndez Reyes (Secretario General de ambas asociaciones) } \\
\text { ÁMBITO ACTUACIÓN: Representa el sur de provincia Alicante con } \\
\text { AICE y Nacional con FICE. } \\
\text { No ASOCIADOS: } 230 \text { empresas asociadas en AICE. }\end{array}$ \\
\hline 3.- CERÁMICA & $\begin{array}{l}\text { ASCER (Asociación Española de Fabricantes de Azulejos y Pavimentos } \\
\text { Cerámicos) } \\
\text { Dña. Ana Martínez (Responsable Comunicación y Prensa) } \\
\text { ÁMBITO ACTUACIÓN: Nacional. } \\
\text { No ASOCIADOS: } 200 \text { empresas asociadas (de las que la mayoría } \\
\text { están concentradas en la Comunidad Valenciana). }\end{array}$ \\
\hline 4.- ILUMINACIÓN & $\begin{array}{l}\text { ARVET (Agrupación de Exportadores de Transformados - Aparatos } \\
\text { Iluminación) } \\
\text { FEDAI (Federación Española de Asoc. de Industriales y Exportadores } \\
\text { de Aparatos de Iluminación) } \\
\text { D. Vicente Martínez (Director de ARVET) } \\
\text { Dña. Ma Ángeles Muñoz (Directora de FEDAI) } \\
\text { ÁMBITO ACTUACIÓN: ARVET Com. Valenciana y FEDAI nacional. No } \\
\text { ASOCIADOS: } 100\end{array}$ \\
\hline 5.- JUGUETE & $\begin{array}{l}\text { AEFJ (Asociación Española de Fabricantes de Juguetes) } \\
\text { D. José Antonio Pastor (Director General Adjunto) } \\
\text { ÁMBITO ACTUACIÓN: Nacional. }\end{array}$ \\
\hline
\end{tabular}




\begin{tabular}{|l|l|}
\hline 6.- MUEBLE & $\begin{array}{l}\text { No ASOCIADOS: 100 empresas asociadas (de las que la mayoría } \\
\text { están concentradas en la Comunidad Valenciana). }\end{array}$ \\
\hline ANIEME (Asociación Nacional de Industriales y Exportadores de \\
Muebles de España) \\
Dña. Amparo Bertomeu (Directora General) \\
ÁMBITO ACTUACIÓN: Nacional \\
No ASOCIADOS: 250 empresas asociadas (de las que la mayoría \\
están concentradas en la Comunidad Valenciana). \\
\hline 7.- TEXTIL & $\begin{array}{l}\text { ATEVAL (Asociación de Empresarios Textiles de la Comunidad } \\
\text { Valenciana) } \\
\text { D. José Serna (Secretario General) } \\
\text { ÁMBITO ACTUACIÓN: Comunidad Valenciana. } \\
\text { No ASOCIADOS: } 530 \text { empresas asociadas. }\end{array}$ \\
\hline
\end{tabular}

Elaboración propia

-2a Muestra, trabajo de campo II. Selección de cuatro empresas de cada uno de los siete sectores.

La segunda muestra para la realización del trabajo de campo II se obtiene en las entrevistas con los Secretarios Generales o Directores Generales de las asociaciones entrevistadas. Son ellos o ellas los que indican una serie de empresas de su sector que presentan un grado más alto de desarrollo en la gestión de comunicación corporativa. A partir de ese listado concreto, se selecciona por disponibilidad de sus directivos y profesionales las cuatro empresas que representan al sector. Con todos estos condicionantes, la muestra definitiva del trabajo de campo II aparece reflejada en la siguiente tabla.

Tabla II. Muestra trabajo de campo II.

\begin{tabular}{|l|l|}
\hline SECTOR EMPRESARIAL CV & EMPRESA ENTREVISTADA \\
\hline 1.- AGROALIMENTACIÓN & $\begin{array}{l}\text { (1) NICOLAU JAMONES } \\
\text { D. Enrique Nicolau (Gerente y Director de Producción y Calidad) }\end{array}$ \\
\cline { 2 - 2 } & $\begin{array}{l}\text { (2) DULCESOL } \\
\text { D. Rafael Juan (Consejero Delegado) }\end{array}$ \\
\hline
\end{tabular}




\begin{tabular}{|c|c|}
\hline & $\begin{array}{l}\text { (3) INTERCITRUS } \\
\text { Dña. Anabel Siguan (Directora Técnica) }\end{array}$ \\
\hline & $\begin{array}{l}\text { (4) CHOCOLATES VALOR } \\
\text { D. Pedro López (Director Gerente) }\end{array}$ \\
\hline 2.- CALZADO & $\begin{array}{l}\text { (1) CALZADOS POLO SUR } \\
\text { Dña. Marian García (Comité Dirección y Dir. Comercial) }\end{array}$ \\
\hline & $\begin{array}{l}\text { (2) PANTER } \\
\text { D. Ramón Pajares (Gerente) }\end{array}$ \\
\hline & $\begin{array}{l}\text { (3) CALZADOS PAKER } \\
\text { D. Salvador Martínez (Gerente) } \\
\text { D. Francisco García (Gerente) }\end{array}$ \\
\hline & $\begin{array}{l}\text { (4) PIKOLINOS } \\
\text { D. Javier Soler (Adjunto Dir. Comercial y Comunicación) }\end{array}$ \\
\hline 3.- CERÁMICA & $\begin{array}{l}\text { (1) CERÁMICA DECORATIVA } \\
\text { D. José Castellano (Director General) }\end{array}$ \\
\hline & $\begin{array}{l}\text { (2) KEROS } \\
\text { D. Joaquín Emilio Martí (Gerente) }\end{array}$ \\
\hline & $\begin{array}{l}\text { (3) GAYA } \\
\text { D. Luigi M. Limido (Director de Marketing) }\end{array}$ \\
\hline & $\begin{array}{l}\text { (4) TAU CERÁMICA } \\
\text { D. Manuel Llopis (Director de Comunicación) }\end{array}$ \\
\hline 4.- ILUMINACIÓN & $\begin{array}{l}\text { (1) LÁMPARAS RAFAEL TORMO } \\
\text { Dña. Amparo Tormo (Gerente) }\end{array}$ \\
\hline & $\begin{array}{l}\text { (2) SAVOY HOUSE } \\
\text { D. Francisco Albiñana (Director General) }\end{array}$ \\
\hline & $\begin{array}{l}\text { (3) ANTARES } \\
\text { D. Guillermo Simó (Director de Marketing) }\end{array}$ \\
\hline
\end{tabular}




\begin{tabular}{|c|c|}
\hline & $\begin{array}{l}\text { (4) ALMERICH } \\
\text { D. Antonio Almerich (Director Gerente) }\end{array}$ \\
\hline \multirow[t]{4}{*}{ 5.- JUGUETE } & $\begin{array}{l}\text { (1) MINILAND } \\
\text { D. José Verdú (Director General) }\end{array}$ \\
\hline & $\begin{array}{l}\text { (2) MUÑECAS ARIAS } \\
\text { D. Francisco Arias (Gerente) }\end{array}$ \\
\hline & $\begin{array}{l}\text { (3) JUGUETES FALOMIR } \\
\text { D. José María Castellano (Director Comercial) }\end{array}$ \\
\hline & $\begin{array}{l}\text { (4) FÁBRICA DE JUGUETES CHICOS } \\
\text { D. Emilio Valls (Director Comercial) }\end{array}$ \\
\hline \multirow[t]{4}{*}{ 6.- MUEBLE } & $\begin{array}{l}\text { (1) COLECCIÓN ALEXANDRA } \\
\text { D. Juan Ventura (Gerente) }\end{array}$ \\
\hline & $\begin{array}{l}\text { (2) CASANOVA GANDÍA } \\
\text { D. Pedro Casanova (Gerente) }\end{array}$ \\
\hline & $\begin{array}{l}\text { (3) PALANCA } \\
\text { D. José Carlos Palanca (Gerente) }\end{array}$ \\
\hline & $\begin{array}{l}\text { (4) ANDREU WORLD } \\
\text { D. José Palau (Director de Marketing y Ventas) }\end{array}$ \\
\hline \multirow[t]{4}{*}{ 7.- TEXTIL } & $\begin{array}{l}\text { (1) REVERT } \\
\text { D. José Ramón Revert (Gerente) }\end{array}$ \\
\hline & $\begin{array}{l}\text { (2) BELPLA } \\
\text { D. José Belda (Gerente) }\end{array}$ \\
\hline & $\begin{array}{l}\text { (3) DOLORES CORTÉS } \\
\text { Dña. Dolores Font Cortés (Gerente) }\end{array}$ \\
\hline & $\begin{array}{l}\text { (4) AZNAR TEXTIL (BONDRAP) } \\
\text { Dña. Ana Aznar (Comité de Dirección y Dir. Marketing y } \\
\text { Comunicación) }\end{array}$ \\
\hline
\end{tabular}


Trabajo de campo III

El siguiente paso metodológico pretende ser una aproximación al mismo objeto desde la visión de los agentes externos que colaboran en la gestión de la imagen y la comunicación de estos sectores, los profesionales de la comunicación valenciana, persiguiendo como principal objetivo la validación y contraste de los resultados del trabajo de campo I y II.

Han sido realizados dos grupos focales, con representantes de las dos Juntas de Gobierno de los dos máximos órganos de representación de la profesión de la comunicación valenciana.

- Ilustre Colegio Oficial de Publicitarios y Relaciones Públicas de la Comunidad Valenciana.

- Asociación de Agencias de Publicidad de la Comunidad Valenciana (AAPCV).

El listado de participantes y cargos que tienen se enumera a continuación:

El primer grupo focal fue realizado el 29 de junio de 2009, en la propia sede de la AAPCV. Los componentes de la junta de gobierno que asistieron fueron los que se especifican:

1.- D. Eugenio Martín, Presidente del Grupo Cuestión.

2.- Dña. Carlota Serratosa, Consejera Delegada de Dimarco.

3.- D. Vicente Vañó, Director de Servicios al Cliente de Engloba.

4.- D. Eduard Farràn, Director Creativo Ejecutivo de Dimarco.

El segundo fue realizado el 13 de julio de 2009, en la sede de Colegio Oficial de Publicitarios y Relaciones Públicas de la Comunidad Valenciana. Con la participación de los siguientes componentes de la junta de gobierno:

1.- Dña. Ana Portaceli, Socia de NGT y Presidenta del Colegio de Publicitarios.

2.- D. Antonio Xandri, Secretario General del Colegio Oficial de Publicitarios.

3.- Dña. Carlota de Dios, Directora General de Carlota de Dios y Asociados.

4.- D. José Segura, Director de Planificación Estratégica de Pértiga Branding.

5.- Dña. Isabel de Salas, profesora de la Universidad CEU Cardenal Herrera.

6.- Dña. Marta Giner, Jefa Comercial y de Relaciones Institucionales de Aumar.

7.- D. Miguel Lucas, Director General de Método y Asociados.

\section{Resultados de la investigación}

El análisis profundo de las distintas etapas de la investigación deriva en la fijación de veinte palancas de cambio -asociadas a las dimensiones micro, meso y macro- que propicien el necesario desarrollo en los sectores tradicionales en materia de comunicación corporativa: 
A) Nivel Micro

1.- Fomento de la elaboración de planes estratégicos empresariales.

Habría que empezar por los cimientos empresariales, ya que si hay empresas que no tienen proyecto empresarial evidentemente actúan y piensan en el corto plazo y por tanto, son incapaces de reaccionar ante la coyuntura actual de crisis. Lógicamente es natural que si no tienen plan empresarial, tampoco tengan planificación alguna de su comunicación, ya que la estrategia de imagen corporativa y de marca debería estar integrada dentro de la estrategia global de la compañía.

2.- Fomento del cambio de mentalidad de "producto fabricado en una empresa" a "empresa que fabrica un producto".

Este cambio de mentalidad enfocado a la corporación y no tanto al producto, ya lo sugería el consultor venezolano Ítalo Pizzolante en una conferencia sobre el marketing del sector cerámico en el año 2007. Se confirma que es un cambio fundamental para darle un enfoque nuevo a la empresa que le haga valorar su imagen corporativa como clave de su competitividad empresarial.

3.- Análisis de las claves de las empresas con éxito empresarial de estos sectores y sus referentes italianos.

Es importante desarrollar estrategias de benchmarketing para estos sectores, con el fin de poder conocer y adoptar aquellas medidas de éxito empresarial de sus competidores, evidentemente siempre adaptadas a las características particulares de las empresas y buscando la diferenciación. Quizá esta también se convertiría en una fórmula para darse cuenta, que sus empresas de referencia llevan años apostando por la marca y no tanto por la producción, que actualmente se ha convertido en un elemento menos determinante, pues se puede fabricar con calidad en cualquier lugar del mundo.

4.- Fomentar la formación del empresario en materia de comunicación.

Se debería estudiar quien es el organismo idóneo para ello y establecer las prioridades formativas tratar. Respecto a la organización, además de las que representan al propio sector profesional de la comunicación también cabría estudiar la implicación de la CIERVAL (patronal autonómica valenciana) y la Universidad, por su carácter más objetivo e imparcial de cara a los empresarios. Respecto a los contenidos deben sentar las bases de la comunicación corporativa y qué puede reportar la consecución de una imagen positiva a su competitividad empresarial. 
5.- Potenciar la introducción de licenciados en publicidad y relaciones públicas, en las áreas de comunicación.

Es cierto que cuantos más licenciados salgan de la universidad y más introducidos estén en las empresas, más opciones habrá de desarrollo en la materia. Pero lo cierto es que a día de hoy se trata de puestos que, o no se han creado o si están introducidos en las empresas no están demasiado bien considerados. Para ello es fundamental formar al alumno con este perfil y potenciar sus capacidades directivas y estratégicas, y no tanto, las ejecutivas.

6.- Potenciar la incorporación de nuevas tecnologías en la actividad comercial de los sectores tradicionales para potenciar la eficacia de la gestión y la imagen de modernidad.

También estas empresas en su labor comercial deberían aprovechar las nuevas tecnologías, pues esto facilitaría no sólo su labor sino la calidad del servicio al cliente y su imagen frente a este, además de su capacidad de segmentación. Las nuevas tecnologías ERP (entreprise resource planning o sistemas de planificación de los recursos) permiten personalizar la oferta para cada cliente, conocer en tiempo real las existencias, los plazos de entrega, etc. En definitiva, implantar la TIC para favorecer una gestión más eficiente.

7.- Creación de una metodología específica para la gestión de la comunicación corporativa en los sectores objeto de estudio.

Aunque las referencias en materia de comunicación corporativa apuestan por un mismo modelo de gestión independientemente del tamaño o tipología de anunciante, sí se deberían estudiar las particularidades que tendría la aplicación del mismo a estos sectores. Temas como el orden de prioridades a establecer, los programas de comunicación corporativa que se deberían ir abordando en función de los recursos y necesidades de este tipo de empresas, las herramientas de investigación, los perfiles profesionales involucrados, los públicos, etc. deberían ser revisados y adaptados a este perfil de empresa. La pyme, con recursos más limitados, necesita ser mucho más selectiva a la hora de establecer prioridades.

8.- Estudiar la manera de dotar de contenido a las marcas de estos sectores empresariales.

A modo de storytelling se debería estudiar cómo definir las marcas de estos sectores, ponerles cara, nombre y personalidad, etc. Con esto se podría alcanzar el modo de sobresalir y diferenciarse de la competencia con personalidad propia.

B)Nivel Meso

9.- Estudio de la viabilidad de la unión de varias empresas para la contratación de servicios de comunicación. 
En uno de los sectores estudiados se ha sugerido esta opción; varias empresas de productos complementarios, estaban estudiando la posibilidad de unirse para la contratación de los servicios del área de comunicación y poder de este modo asumir los costes. No obstante, se trata de una intención no materializada a día de hoy, ni de la que se hayan obtenido resultados hasta la fecha pero se podría estudiar como fórmula para la introducción de políticas de comunicación en este perfil de empresas. Otra opción a estudiar sería en vez de la contratación de servicios externos de comunicación, la contratación de un director de comunicación, que trabaje para varias empresas de sectores complementarios.

10.- Viabilidad de establecer una asociación de pequeños anunciantes o extensión de la asociación española de anunciantes (AEA).

Otra de las futuras líneas de trabajo podría ser el estudio de qué puede aportar al desarrollo de la comunicación en la comunidad, la creación de la Asociación de Pequeños Anunciantes o incluso de Anunciantes de la Comunidad Valenciana. Al igual que determinar quién debería componerla, cuáles serían sus objetivos y actividades, etc. Las actividades de la AEA son muy interesantes para potenciar el sector, pero están centradas exclusivamente en grandes compañías, lo que hace que el perfil de las empresas de los sectores tradicionales no se sientan representadas. Se trataría de adaptar el discurso a las necesidades y al lenguaje de este perfil de empresas.

11.- Construcción de una reputación sectorial y una reputación empresarial simultáneamente.

Quizá haya que trabajar a dos bandas en la construcción de una reputación corporativa para estos sectores, por un lado, de forma sectorial a través de las asociaciones empresariales construyendo una imagen fuerte para cada uno de los sectores o para todos los sectores tradicionales en su conjunto. $\mathrm{Y}$ al mismo tiempo construyendo una imagen corporativa individual para cada una de las empresas a través de la gestión de la comunicación corporativa, la comunicación interna y la de producto. En este caso las asociaciones deben jugar un papel más relevante todavía, en la creación de marca sector.

12.- Análisis de la idoneidad de crear marcas territorio para potenciar estos sectores empresariales.

Es cierto que se trata de clusters muy concentrados geográficamente y esto podría ser una oportunidad para el desarrollo de marcas territorio que dotaran de valor a la marcas empresariales.

13.- Potenciar las marcas creadas por las asociaciones a modo de distintivos de calidad. 
Marcas o campañas como Éthica-tex, del sector textil, Fecha Cierta, del sector juguete, etc. son iniciativas que deberían replantearse y buscar más apoyos de los mismos sectores de otros territorios o incluso de otros sectores con la misma problemática, incluso que tuvieran trascendencia más allá del territorio nacional, etc. con la intención de coger fuerza y protagonismo. Son iniciativas interesantes que no han prosperado demasiado, por falta de recursos al tratarse de proyectos circunscritos en un ámbito pequeño como pueden ser las asociaciones empresariales de estos sectores tradicionales.

14.- Desarrollo de grupos de innovación para establecer nuevos modelos de negocio y nuevas vías de desarrollo.

Se trata de potenciar la creatividad de estos empresarios para la resolución de problemas planteados a raíz de la crisis, uno de los más relevantes es la bajada de la demanda, la falta de competitividad, etc. y por ello, este podría ser uno de los muchos temas a tratar. Esta iniciativa consistiría en luchar contra la paralización generalizada que ha sido provocada por la situación de crisis y potenciada, por el talante empresarial de estos sectores tradicionales.

C)Nivel Macro

15.- Establecer fórmulas para fomentar el conocimiento y a su vez, la confianza, entre profesionales de la comunicación y anunciante.

Esta es una problemática que deben resolver las dos organizaciones que representan al sector de la comunicación valenciana, la AAPCV y el Ilustre Colegio Oficial de Publicitarios y Relaciones Publicas de la Comunidad Valenciana. Sendas organizaciones tienen el deber y la responsabilidad, principalmente por su beneficio profesional, de resolver la confusión que tienen los empresarios sobre qué tipo de servicios ofrece la profesión, qué tipo de tarifas se pueden esperar, los distintos perfiles de agencias y la actividad de cada una de ellas.

16.- Estudiar la posibilidad de poner en marcha una campaña de prestigio y reposicionamiento de los profesionales de la comunicación valencianos.

La falta de credibilidad de la profesión frente a los empresarios era un tema preocupante y que paraliza la posibilidad de introducción de políticas de comunicación en las empresas. Para frenar esta tendencia se debe poner en valor y prestigiar la profesión frente a los anunciantes, además de luchar para poder diferenciarse del pseudosector que tanto daño hace a nivel de imagen y confianza.

17.- Investigar hacia dónde debe ir dirigido el nuevo modelo de negocio de las agencias de publicidad valencianas. 
Las agencias deberían también replantearse su modelo de negocio puesto que en estos momentos, estos sectores están faltos de consultores de comunicación estratégica o de departamentos externos de comunicación que les ayuden a planificar estratégicamente, y a través de un trabajo en conjunto. Esto es algo que la mayoría de agencias y de profesionales no está ofreciendo a sus clientes pero porque la mayoría no sabe cómo hacerlo, ya que hay una falta de profesionales expertos en consultoría. Esta línea de negocio, además no es tan sensible al precio.

18.- Estudio de cómo potenciar los medios no convencionales para estos sectores.

Los medios interactivos y de contacto personalizado, como técnicas de CRM, son medios con gran eficacia para estos sectores y sobre todo tarifas muy asequibles, se debería por tanto estudiar, cuáles son los más idóneos, cómo utilizarlos y cómo potenciarlos.

19.- Elaboración de tarifas propias de cada agencia y de sus servicios de comunicación, a favor de la transparencia.

Con el fin de poder reposicionar la imagen del sector de la comunicación, habría que potenciar y poner en marcha, el que cada agencia elaborara sus tarifas según servicios ofertados, para que sirvan de referencia para el anunciante. Incluso estudiar la opción de establecer en sus honorarios cierta variable, en base a objetivos medibles alcanzados (de comunicación), de esta manera, se involucra el trabajo de la agencia económicamente en los resultados, lo que redundaría en credibilidad y confianza.

20.- Soluciones ad hoc para estos sectores a la hora de medir la eficacia de la comunicación.

Desde la universidad, junto con las agencias y los anunciantes se deben buscar también soluciones ad hoc para medir la eficacia de las acciones de comunicación, está claro que ya existen fórmulas de pre-test y de post-test, pero estas, deben adaptarse a las necesidades y recursos de estos sectores. Es vital demostrar que la comunicación funciona.

\section{Conclusiones}

Las conclusiones de esta investigación o claves para el desarrollo de la comunicación corporativa en los sectores tradicionales valencianos se agrupan en torno a tres niveles o dimensiones:

El primero de ellos es el nivel micro o interno de las propias empresas. Es imprescindible que estas organizaciones asuman un proyecto empresarial basado en el largo plazo y que integre a todas las áreas y funciones, no solo a comunicación. De forma paralela, deberá instalarse una concepción corporativa de la organización más allá de autoimagen de fabricante. Para 
ello será imprescindible la formación en comunicación de los líderes de estas empresas. La evolución exige el empleo de distintas técnicas y herramientas como la investigación, las nuevas tecnologías o el storytelling vinculado a marcas así como de metodologías específicas del área de comunicación corporativa. La penetración de profesionales de comunicación en sus respectivas plantillas será determinante.

El segundo ámbito es el sectorial o nivel meso. En este plano la cooperación entre distintas empresas de un mismo sector puede redundar en un notable salto cualitativo en materia de comunicación. Las fórmulas o acciones son diversas: asociaciones de pequeños anunciantes para defender sus intereses, definición de marcas territorio vinculadas a los clusters, marcas de las distintas asociaciones como sellos de calidad, planificación de una reputación sectorial, grupos de innovación vinculados a la práctica de comunicación. En definitiva, medidas que pongan en valor la potencia de una marca/territorio/sector ante una público de carácter global.

Finalmente, el tercer nivel es el macro, el ámbito más allá de la empresa y el sector que en este caso tiene que ver con sus proveedores específicos de comunicación. En esta relación determinante para esta evolución - es necesario una mayor confianza o fijación de un status de partner. Habría que incidir en el modelo de remuneración de las agencias y en la medición de la eficacia de las distintas campañas de comunicación. Las agencias a su vez también deberían realizar un esfuerzo para adaptarse a las necesidades del cliente, tanto en su modelo de negocio como en los servicios que presta reorientándolos hacía la planificación estratégica. A su vez agencias y proveedores también deberían poner en valor o prestigiar su oficio frente a los propios sectores tradicionales para favorecer su credibilidad.

\section{REFERENCIAS BIBLIOGRÁFICAS}

ALVAREZ-GAYOU JURGENSON, J. L (2003): Cómo hacer una investigación cualitativa, fundamentos y metodología, México: Paidós Ecuador,

BEL MALLÉN, J. I. (coord.). (2004): Comunicar para crear valor. Dirección de comunicación en las organizaciones. Pamplona: EUNSA.

BENLLOCH OSUNA, M,T. Y LÓPEZ LITA, R. (2005): La marca territorio. El marketing de ciudad, una herramienta al servicio de las marcas territorio. Castellón: Ed. Grupo Mibalia. Publicación: 99\%.com ISSN: 1885-4397.

BENLLOCH OSUNA, M,T. Y LÓPEZ LITA, R. (2006): De la marca comercial a la marca territorio. Revista Recerca. Castellón:. Revista de Pensament i Anàlisi . ISSN: 11306149.

BLAY ARRÁEZ, R. Y BENLLOCH OSUNA, M,T. (2006): ¿Qué puede hacer la comunicación por el sector? Castellón: Periódico El mundo, Castelló al día. Especial Cevisama. 
BLAY ARRÁEZ, R. Y BENLLOCH OSUNA, M,T. (2007): Análisis de la comunicación en los sectores relevantes para la provincia de Castellón. Castellón: Ed. Grupo Mibalia. Publicación: 99\%.com ISSN: 1885-4397.

BLAY ARRÁEZ, R. Y BENLLOCH OSUNA, M,T. (2010): "La gestión de la imagen y la comunicación corporativa en las pequeñas y medianas empresas valencianas" en Lopéz Lita, R y Mut Camacho, M. (Coord) Gestión de intangibles. Importancia en el desarrollo territorial. Castellón: Ed. Ciencias Sociales.

CÁMARA OFICIAL DE COMERCIO, INDUSTRIA Y NAVEGACIÓN DE VALENCIA (2004): "La nueva economía global y su incidencia en los sectores tradicionales de la Comunidad Valenciana", Valencia: Cámara de Comercio en febrero de 2004.

CAPRIOTTI, P. (2009): Branding corporativo.Fundamentos para la gestión estratégica de la identidad corporativa. Santiago de Chile: Libros de Empresa.

CARRILLO, M.V. y otros. (2005): Imagen y comunicación en las pymes. Madrid: Editorial Fragua.

COSTA, J. (2003): Imagen corporativa en el siglo XXI. Buenos Aires: La Crujía.

COSTA, J. (2004): La imagen de marca. Un fenómeno social. Barcelona: Paidós.

COSTA, J. (2008): El dircom hoy. Barcelona:Costa Punto Com Editor.

GARCÍA OROSA, B. (2005): Los altavoces de la actualidad: radiografía de los gabinetes de comunicación. Netbiblo.

GARRIDO, F. J. (2001): Comunicación estratégica. Las claves de la comunicación empresarial en el siglo XXI. Barcelona: Gestión 2000.

GENERALITAT VALENCIANA (2009): "II Planes sectoriales de competitividad", nota de prensa emitida por el IMPIVA el 5 de enero de 2009, Valencia. Fecha consulta: 5 septiembre de 2009. www.impiva.es.

LÓPEZ LITA, R. (2000): La comunicación: la clave del bienestar social. Madrid: Ediciones El Drac.

LÓPEZ LITA, R. (2001): Las agencias de publicidad: evolución, posicionamiento y futuro. Castellón: Publicaciones de la Universitat Jaume I.

PÉREZ GONZÁLEZ, R.A. (2006): Estrategias de comunicación. Barcelona: Ariel.

PIZZOLANTE NEGRÓN, I. (2006): El poder de la comunicación estratégica. Apuntes de un evangelizador corporativo. Venezuela: El Nacional.

PLA BARBER, J. y otros (2007): "Crisis, actitudes directivas y estrategia en los sectores manufactureros tradicionales: el sector textil español". Madrid: Universia Business Review - Actualidad Económica, segundo trimestre 2007.

VILLAFAÑE, J. (1999): La gestión profesional de la imagen corporativa. Madrid: Pirámide VILLAFAÑE, J. (2004): La buena reputación. Madrid: Pirámide

[Recibido: 7 de noviembre de 2013. Aceptado con cambios: 15 de septiembre de 2014. Aceptación definitiva: 14 de octubre de 2014] 